\title{
Putative, phenylacetaldehyde synthase transcript of vanda mimi palmer: sequence and expression analysis.
}

\begin{abstract}
Vanda Mimi Palmer is a fragrant-orchid of which has won several international awards for its fragrance emission characteristic. A partial sequence encoding putative phenylacetaldehyde synthase (VMPPAAS) transcript which might be involved in the fragrance biosynthesis of this orchid was previously identified in the floral Expressed Sequence Tags (ESTs) of this orchid. The aims of this study were to isolate full-length cDNA of VMPPAAS and followed by molecular characterization. The full-length cDNA was isolated by SMART RACE PCR amplification while the expression analysis was carried out using real-time RT-PCR for different tissues, different flower developmental stages and different time points in a $24 \mathrm{~h}$ cycle. The VMPPAAS transcript encodes for a polypeptide of 508 amino acid residues consisting of 1524 bp Open Reading Frame (ORF) flanked by 92 bp of 5' Untranslated Region (UTR) and $93 \mathrm{bp}$ of 3' UTR including a poly-A tail. It has a conserved motif for pyridoxal phosphate attachment site and a VHVDAAY motif, which are also shared by other plant decarboxylase proteins such as tyrosine decarboxylases, tryptophan decarboxylases and phenylacetaldehyde synthase. Expression analysis of VMPPAAS transcript by real-time RTPCR showed an up-regulated expression in floral tissues especially in sepal and petal and down-regulated in vegetative tissues. The expression was shown to be developmentally regulated in the flower life-cycle as well as temporally regulated in a $24 \mathrm{~h}$ cycle.
\end{abstract}

Keyword: Fragrant-orchid; Phenylacetaldehyde synthase; VMPAAS; Molecular chareacterization; Real-time RTPCR; Fragrance genes; Orchid hybrid. 